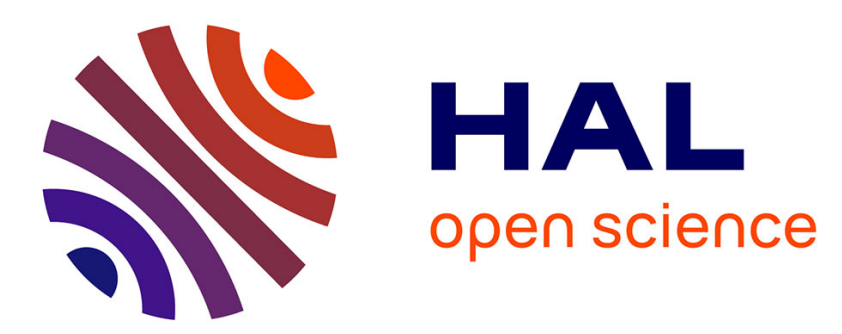

\title{
Nouvelles découvertes de peintures rupestres dans la Corne de l'Afrique: les abris sous roche de Las Geel, République de Somaliland
}

Xavier Gutherz, Joséphine Lesur, Jean-Paul Cros

\section{- To cite this version:}

Xavier Gutherz, Joséphine Lesur, Jean-Paul Cros. Nouvelles découvertes de peintures rupestres dans la Corne de l'Afrique: les abris sous roche de Las Geel, République de Somaliland. Annales d'Éthiopie, 2003, XIX, pp.295-306. hal-02155258

\section{HAL Id: hal-02155258 \\ https://hal.science/hal-02155258}

Submitted on 13 Jun 2019

HAL is a multi-disciplinary open access archive for the deposit and dissemination of scientific research documents, whether they are published or not. The documents may come from teaching and research institutions in France or abroad, or from public or private research centers.
L'archive ouverte pluridisciplinaire HAL, est destinée au dépôt et à la diffusion de documents scientifiques de niveau recherche, publiés ou non, émanant des établissements d'enseignement et de recherche français ou étrangers, des laboratoires publics ou privés. 


\title{
Persée
}

http://persee2.univ-lyon2.fr

\section{Nouvelles découvertes de peintures rupestres dans la Corne de l'Afrique : les abris sous roche de Las Geel, République de Somaliland}

\author{
Xavier Gutherz;Joséphine Lesur;Jean-Paul Cros \\ Annales d'Ethiopie, Année 2003, Volume 19, Numéro 1 \\ p. $295-306$
}

Voir l'article en ligne

\section{Avertissement}

L'éditeur du site «PERSEE » - le Ministère de la jeunesse, de l'éducation nationale et de la recherche, Direction de l'enseignement supérieur, Sous-direction des bibliothèques et de la documentation - détient la propriété intellectuelle et les droits d'exploitation. A ce titre il est titulaire des droits d'auteur et du droit sui generis du producteur de bases de données sur ce site conformément à la loi n $98-536$ du 1er juillet 1998 relative aux bases de données.

Les oeuvres reproduites sur le site «PERSEE » sont protégées par les dispositions générales du Code de la propriété intellectuelle.

Droits et devoirs des utilisateurs

Pour un usage strictement privé, la simple reproduction du contenu de ce site est libre.

Pour un usage scientifique ou pédagogique, à des fins de recherches, d'enseignement ou de communication excluant toute exploitation commerciale, la reproduction et la communication au public du contenu de ce site sont autorisées, sous réserve que celles-ci servent d'illustration, ne soient pas substantielles et ne soient pas expressément limitées (plans ou photographies). La mention Le Ministère de la jeunesse, de l'éducation nationale et de la recherche, Direction de l'enseignement supérieur, Sous-direction des bibliothèques et de la documentation sur chaque reproduction tirée du site est obligatoire ainsi que le nom de la revue et- lorsqu'ils sont indiqués - le nom de l'auteur et la référence du document reproduit.

Toute autre reproduction ou communication au public, intégrale ou substantielle du contenu de ce site, par quelque procédé que ce soit, de l'éditeur original de l'oeuvre, de l'auteur et de ses ayants droit.

La reproduction et l'exploitation des photographies et des plans, y compris à des fins commerciales, doivent être autorisés par l'éditeur du site, Le Ministère de la jeunesse, de l'éducation nationale et de la recherche, Direction de l'enseignement supérieur, Sous-direction des bibliothèques et de la documentation (voir http://www.sup.adc.education.fr/bib/). La source et les crédits devront toujours être mentionnés. 


\title{
NOUVELLE DÉCOUVERTE DE PEINTURES RUPESTRES DANS LA CORNE DE L'AFRIQUE: LES ABRIS SOUS-ROCHE DE LAS GEEL, RÉPUBLIQUE DE SOMALILAND
}

\author{
Xavier Gutherz', Jean-Paul Cros $^{2}$ et Joséphine Lesur ${ }^{3}$
}

\section{Circonstances de la découverte}

C'est dans le cadre d'une mission de prospection archéologique réalisée en novembre et décembre 2002 en République de Somaliland ${ }^{4}$ que nous avons été conduits le 4 décembre sur le site de Las Geel, alors que nous rentrions à Hargeisa en fin de mission. Ce site nous a été indiqué par un habitant du village de Daarbudhuq, une localité située sur la route nationale entre Hargeisa et Berbera à mi-chemin entre les deux villes. Cette personne nous a conduits sur le site.

Le petit massif de granite rouge de Las Geel se trouve à 4 $\mathrm{km}$ à l'ouest de la route d'Hargeisa à Berbera. Ce massif rocheux se détache d'un vaste plateau granitique surcreusé par des oueds (Figure 1). Au pied du rocher de Las Geel se trouve le confluent de deux oueds. Le nom du lieu s'explique aisément par la présence de laisses d'eau temporaires et d'une nappe permanente proche de la surface du lit

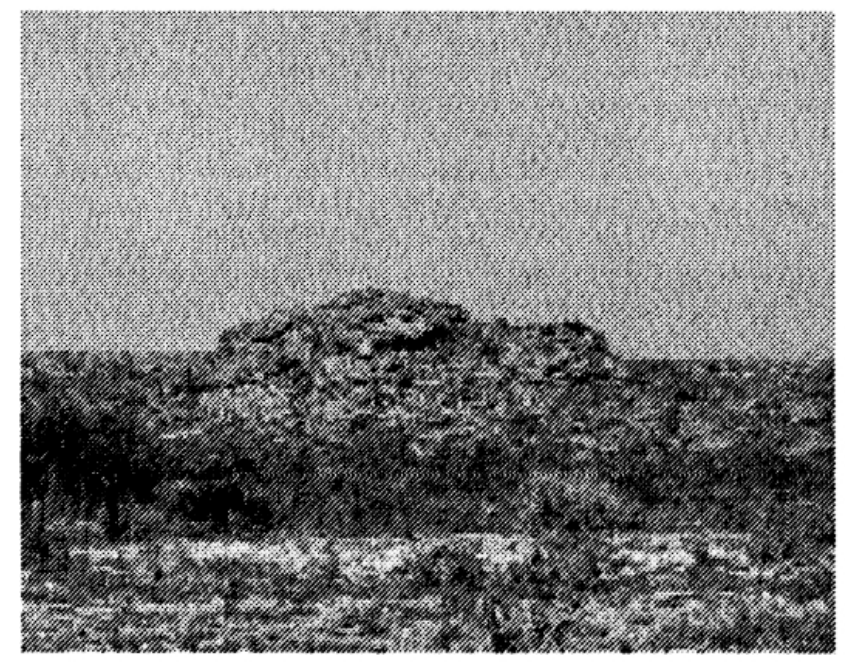

Vue générale du site de Las Geel des oueds. En effet, ce nom signifie en langue somali : "le point d'eau des dromadaires". Actuellement, à la dénomination de Las Geel s'est substituée celle

\footnotetext{
${ }^{1}$ Chef de la mission, professeur à l'université Paul Valéry-Montpellier III et directeur adjoint de I'UMR 6636 (ESEP - MMSH, Aix-en-Provence).

2 Docteur en médecine, paléoanthropologue, chercheur associé à l'UMR 7041, Nanterre (équipe Afrique, sociétés et environnement)

${ }^{3}$ Doctorante à l'université de Paris 1, et ESA 8045 Archéozoologie et histoire des sociétés, MNHN, Paris.

${ }^{4}$ Cette mission a été réalisée grâce à une allocation de recherche du Ministère Français des Affaires Etrangères et grâce à la bienveillance des autorités somalilandaises, en particulier M. Osman Ali Bile, ministre de la culture et du tourisme que nous tenons à remercier chaleureusement ainsi que ses collaborateurs. Nos remerciements vont également à Mohamed Omar Ismaël et à Mohamed Abdi Ali pour leur précieux concours sur le terrain.
} 
de Las Gaal, mais nous avons préféré sur les conseils de nos accompagnateurs somalilandais revenir à l'orthographe d'origine qui a une signification plus claire.

\section{Description physique}

Le rocher de Las Geel comporte une vingtaine d'abris de taille modeste ouverts sur tout son pourtour. Les peintures rupestres ne sont présentes que dans les abris du versant oriental. Sur le flanc sud, existent cependant quelques traces très effacées, alors que les quelques surplombs du versant occidental ne semblent pas avoir abrité de peintures. En revanche, toute la périphérie du massif rocheux est jonchée d'industrie lithique (éclats, nucleus, pièces façonnées en silex) sur laquelle nous reviendrons plus loin.

Les panneaux peints se trouvent répartis sous une dizaine d'abris dont les plus vastes ont une profondeur ne dépassant pas $5 \mathrm{~m}$ et une largeur pouvant atteindre $10 \mathrm{~m}$. Ces abris naturels creusés par les effets combinés de la thermoclastie, de la corrosion et de l'érosion éolienne sont répartis sur trois niveaux. Le niveau supérieur en comporte 3 séparés par 2 autres ensembles peints, l'un, situé un peu plus haut sous un petit surplomb, l'autre dans une alcôve. Vers le Nord, à quelques dizaines de mètres de ce premier ensemble, sur des parois subverticales, des traces de peinture rouge très délavées attestent la présence ancienne de panneaux ornés; il en est d'ailleurs de même vers le Sud, mais dans ces deux cas, la verticalité des supports et l'absence de surplomb ou leur éboulement ancien n'a pas permis la conservation des peintures.

Le niveau intermédiaire comporte un abri décoré ainsi qu'une alcôve en cloche présentant une thématique particulière que nous décrirons plus loin. Enfin, au niveau le plus bas, les deux derniers abris très richement peints possèdent, du moins pour l'un d'entre eux, un traitement particulier des représentations animales et humaines. Nous n'avons pu observer l'autre qu'à l'aide de jumelles, à une certaine distance car des nids de grosses guêpes très agressives nous en ont interdit l'approche.

\section{Les peintures}

Les représentations animales et humaines dominent largement la thématique de l'ensemble de ces abris, une thématique qui est d'ailleurs commune à tous les panneaux.

Il s'agit d'une combinaison de figures monochromes, les moins nombreuses, et de figures polychromes. Les couleurs utilisées sont par ordre de fréquence, l'ocre rouge, le blanc, l'ocre jaune et plus rarement le noir.

\subsection{Les bovinés}

Les nombreux bovinés figurés à Las Geel sont des vaches dont le pis et les quatre mamelons sont nettement dessinés (Planches 1, 2, 3, 4 \& couverture). 
Ces vaches ont un dos sans bosse. Leur robe est soit unicolore, soit pie (Planche 4). Les longues cornes sont le plus souvent en pince (Planches 2,4 \& couverture), plus rarement en lyre (Planche 1) ou droites et souvent entièrement peintes en blanc. Leurs extrémités sont assez fréquemment peintes en brun rouge. Ces cornes sont parfois ornées de traits rouge ondoyants (Planche 3). Les queues, très courtes, sont rarement représentées. Les pattes ne sont pas différenciées, le corps de l'animal étant figuré de profil. Le plus surprenant est le traitement des cous et des têtes. Les cous sont longs, filiformes, figurés par un trait prolongeant la ligne du dos et ils portent à leur extrémité distale une tête de petite taille en forme de vase à panse galbée ou de cloche (Planches 1, 2, 3, 4 $\&$ couverture). Ces têtes de forme particulière sont vues de dessus. Elles sont quelquefois ornées de petits traits ou de points rayonnants disposés en auréole autour d'elles.

Un simple trait prolonge donc la ligne dorsale jusqu'à la tête qui est détachée de la masse du corps. Au-dessous de ce trait, au niveau du cou, se développe une plage subrectangulaire possédant un rostre arrondi à la base vers l'avant. L'espace intérieur de ce motif subrectangulaire aux angles adoucis est traité de diverses façons : soit il est laissé vide et permet parfois de voir la ligne du cou comme par transparence (Planches $2 \& 3$ ), soit il est peint de manière uniforme (Planche 4) ou encore décoré de bandes verticales (ou très rarement horizontales) jointives et peintes alternativement en deux couleurs (rouge et blanc le plus souvent, noir et blanc dans un cas) (Planches $1,4 \&$ couverture).

Du point de vue de la taille, bien que des mesures précises n'aient pu être effectuées, faute de temps disponible en cette fin de mission, on peut estimer que les bovins ont une longueur moyenne qui se situe entre 40 et $50 \mathrm{~cm}$ pour une hauteur de 20 à $30 \mathrm{~cm}$. Quelques animaux sont de plus petite taille $(20 \mathrm{~cm}$ de long environ).

Bien que chaque panneau comprenne au minimum 3 à 4 animaux, ce nombre pouvant aller jusqu'à une quinzaine pour les panneaux les plus fournis, on ne peut pas considérer qu'il existe de représentation de troupeau, comme c'est souvent le cas dans d'autres sites ornés de la Corne de l'Afrique, à commencer par l'un des plus proches, sur lequel nous reviendrons, l'abri de Kaarin Heegan, situé près de Boosaaso, à $300 \mathrm{~km}$ de Las Geel.

\subsection{Les représentations humaines}

De nombreux anthropomorphes sont peints sur les parois des différents abris mais ils sont cependant moins nombreux que les vaches. Le thorax est large et revêtu d'une sorte de "chemise"

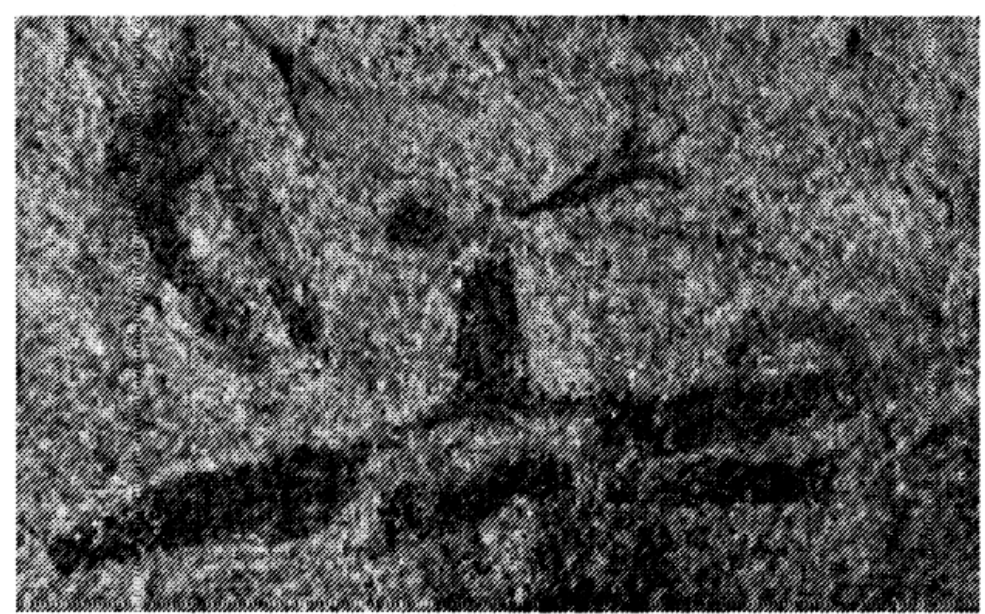

Figure 2 
parfois figurée en bandes peintes verticales et accolées (Planches 1, 3, 4 \& couverture). Les membres inférieurs sont fusiformes, confondus ou séparés par un simple trait ou un espace vertical. Les membres supérieurs sont filiformes et toujours écartés (bras en croix). La tête est de petite taille, punctiforme ou arrondie, ou encore déportée sur un côté et placée au-dessus d'un cou tronconique parfois recoupé de traits horizontaux évoquant des anneaux de collier superposés (Planche de couverture). Cette tête est quelquefois cernée d'une couronne de points ou de petits tirets rayonnants. Dans certains cas, une sorte de plumeau apparaît sur un côté au sommet de la tête (Figure 2). Ces personnages portent quelquefois un arc, un bâton ou éventuellement un petit bouclier. Ils sont systématiquement associés aux bo-vinés et placés soit sous le cou (Planche $3 \&$ couverture), sous le ventre (Planches $1 \& 4$ ) ou plus rarement derrière la croupe. Mis à part le fait qu'ils sont parfois accompagnés de canidés (Planche 4), ces personnages sont représentés sans lien les uns avec les autres et aucune scène d'activité de groupe n'est décelable.

\subsection{Les autres figurations animales}

En dehors des vaches, l'animal le plus représenté est probablement un chien, ou du moins un canidé à queue recourbée et à oreilles distinctes. Ces chiens sont souvent figurés près des personnages (Planche 4 ).

D'autres animaux sont beaucoup plus rarement figurés : Il s'agit de quelques probables antilopes, à l'arrière-train surbaissé et à la ligne de ventre en arceau ( $c f$. gravures de Dorra et Balho à Djibouti) ainsi que de caprinés sur le panneau portant le seule figuration de girafe repérée (Planche 5). Quelques caprinés sont disséminés ça et là sur les autres panneaux.

\subsection{Signes}

Ces abris comportent aussi des signes peints non figuratifs ou difficilement interprétables.

Des ensembles de points ou de petits traits sont associés aux bovinés et aux humains. Ils sont situés en "couronne" autour de la tête de certains animaux mais aussi autour de celle de personnages. Dans une petite alcôve aux parois concaves, une scène unique représente un alignement de 5 lignes verticales peintes à l'ocre rouge sans aucun appendice latéral et terminées au sommet par un cercle plein peint en brun-noir (Planche 1), ces "sujets" filiformes sont placés devant et en partie sous le cou d'une vache mais s'agit-il de la représentation stylisée de personnages filiformes comme il en existe dans le Harar? Ici, en tout cas ni les membres supérieurs ni les membres inférieurs ni le sexe ne sont figurés. On a pu observer que le cou de la vache porte un magnifique décor de bandes verticales blanches alternant avec des bandes brun-noir. Une des bandes blanches porte des taches brun-noir rondes semblables à ces "têtes". Audessous de la vache on trouve un grand personnage, les bras levés et dont le bras gauche semble toucher les 5 mamelons figurés les uns au-dessus des autres. Ya-t-il un lien sémantique entre les 3 sujets ? 
Dans le panneau de l'abri le plus septentrional du niveau inférieur, il existe des "bâtons" allongés et décorés de bandes transversales blanches : l'un au moins porte à son extrémité supérieure une "couronne" de petits traits, ce qui pourrait le faire interpréter comme une figuration humaine très stylisée.

Il existe par endroits - surtout dans l'alcôve du niveau supérieur - des tracés digitaux de couleur rouge ou ocre jaune, superposés ou non aux figurations animales.

Enfin on peut observer d'autres signes ou d'autres figurations difficilement interprétables. Ainsi dans la grande alcôve, des sortes d'épingles à tête annulaire, sur un autre panneau une sorte de "casque" décoré (Figure 3) ou encore un probable animal non identifié qui pourrait être une outarde.

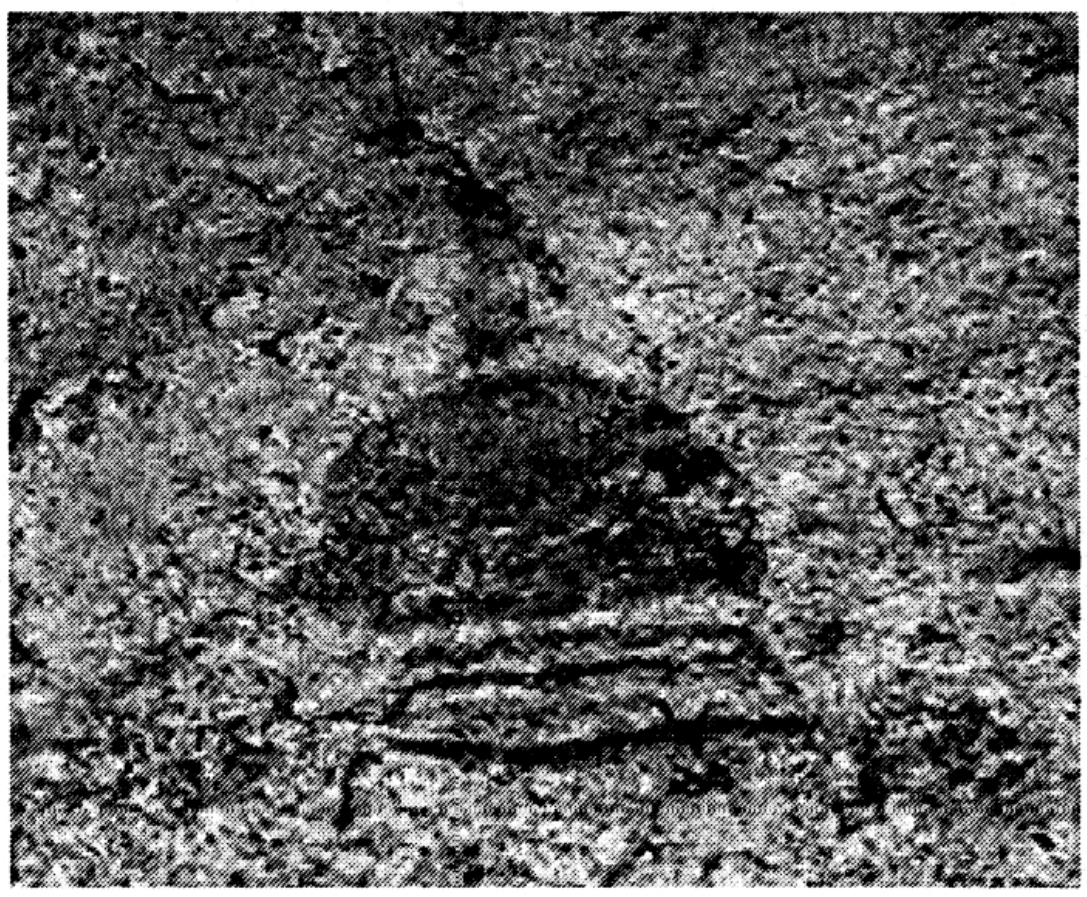

Figure 3

\section{Premières observations d'ordre chronologique et culturel}

\subsection{Questions de chronologie}

D'une façon générale les peintures de Las Geel se rattachent majoritairement à une "période" d'éxécution dont l'unité se reconnaît à travers un style caractéristique. Il existe cependant des cas de superpositions évidentes de phases. C'est notamment le cas sur le grand panneau des bovinés femelles rouges de l'alcôve du niveau supérieur (Planche 2). Sur ce panneau des représentations d'humains ou de bovinés ou de simples traits verticaux au coloris délavé sont recouvertes par les grands bovinés femelles au coloris ocre rouge dont l'état de "fraicheur" est remarquable.

D'autres cas similaires sont visibles çà et là sur le site.

En ce qui concerne la façon de figurer les bovins, certains panneaux permettent d'entrevoir une évolution locale du graphisme depuis un style qui est très 
proche du style arabo-éthiopien du Harar ou de celui de Chabbé-Galma vers le style propre à la phase récente de Las Geel.

Il est bien sûr extrêmement difficile d'assigner un âge précis - dans l'état actuel de l'analyse du site - aux peintures de Las Geel. Lorsque nous aborderons le chapitre des comparaisons, nous verrons qu'en dépit de leur originalité certaine, les peintures se rattachent tout de même à une famille stylistique pan-régionale que l'on a coutume de dater des IIIe et II ${ }^{\mathrm{e}}$ millénaires, période où apparaît et se développe l'élevage bovin dans la Corne de l'Afrique (Joussaume, 1981 et 1989). Les industries lithiques présentes en masse sur le site, sur le sol même de certains abris ou sur les dépôts de pente au pied de ceux-ci devront être analysées mais leur positionnement chronologique ne sera pas aisé. En effet, à première vue, une patine blanche présente sur certaines pièces et absente sur d'autres peut faire penser qu'il existe plusieurs phases d'occupation des lieux, ce qui renvoie peut-être à ce que nous avons pu observer sur les parois peintes. En outre, la classification chrono-typologique des industries lithiques holocènes du Somaliland et de la Grande Somalie n'a pas progressé depuis les travaux de Clark qui remontent aux années 50 et au sujet desquels S. A. Brandt a rappelé plus récemment qu'ils étaient à reprendre entièrement (Brandt, 1986). C'est d'ailleurs un des objectifs que nous nous sommes assigné en entamant un programme de recherche au Somaliland. Le second problème qui se pose ici comme d'ailleurs sur de nombreux sites d'habitat entrevus lors de cette mission, c'est l'absence totale de céramique. Si l'on doit assigner à ces peintures un âge néolithique ou protohistorique, il faudra admettre que des groupes de pasteurs ont pu occuper cette région sans faire usage de la poterie.

On doit mentionner ici les datations très basses obtenues par Brandt sur des charbons de bois prélevés dans un sondage réalisé à l'entrée de l'abri de Karin Heegan (Brandt et al, 1984). Ces dates de 1600 et 2100 BP sont surprenantes si on doit les mettre en relation avec les peintures (bovinés sans bosse, absence de camélidés). Le matériel archéologique trouvé dans le sondage de $2 \mathrm{~m}^{2}$ est très pauvre et non significatif. Il est probable que ces datations correspondent à une occupation postérieure à l'éxécution des peintures.

\subsection{Eléments majeurs de la thématique de Las Ceel}

Ce qui frappe avant tout dans ce riche ensemble de panneaux, c'est la place majeure accordée aux bovinés femelles. Ce sont ces animaux qui ont une taille supérieure à tous les autres animaux mais aussi supérieure aux personnages figurés à leurs côtés. Le traitement très original de l'encolure et de la tête de ces animaux permet d'évoquer une volonté de figuration symbolique qui se distingue d'un dessin réaliste, sauf à considérer qu'il pourrait s'agir de la représentation d'une pièce de tissu ou de cuir posée sur le cou des animaux lors de cérémonies particulières. C'est pourquoi, dans le cas de Las Geel, il est difficile de souscrire au point de vue émis par certains auteurs (Bouakaze-Khan, 2002) qui interprétent les décors sous le cou de bovins comme la figuration des plis graisseux (abri d'Ourso dans le Harar). A Las Geel, cette décoration particulière de l'encolure ajoutée à la représentation schématique de la tête, confère à la figu- 
ration de cette partie du corps une importance particulière dont la signification nous échappe mais qui relève probablement du domaine symbolique. Cela s'oppose d'une certaine façon au réalisme qui caractérise la représentation du pis et qui pourrait suggérer la place importante occupée par le lait dans l'alimentation.

\section{3. Éléments de comparaison}

En ce qui concerne la représentation des humains, on ne peut guère trouver aujourd'hui qu'un seul site de référence dans la région. Il s'agit de l'abri de Karin Heegan, situé à $300 \mathrm{~km}$ au Nord-Est, près de Boosaaso en Grande Somalie (Brandt et Carder, 1987). Dans cet abri richement orné de nombreux bovinés, on observe au moins deux personnages en tous points semblables à ceux de Las Geel. Les autres anthropomorphes de l'ensemble des sites de la Corne de l'Afrique revêtent des aspects divers mais aucun ne peut être assimilé aux figurations de Las Geel.

La façon de représenter les bovins femelles nous ramène en revanche à une ou des ambiances stylistiques connues dans la Corne de l'Afrique, en dehors du traitement très particulier de la tête et du cou. Pour ces parties du corps, là encore, un seul point de comparaison proche peut être évoqué. En effet, à Karin Heegan, un des nombreux bovins possède un plastron orné joignant le poitrail à l'encornure, exactement comme à Las Geel. Ce rapprochement est conforté par la présence à coté de ce bovin du personnage cité ci-dessus. Toutefois, bien que tous les panneaux du site n'aient pas été reproduits dans la publication, il semble bien que cette figure soit une exception, les autres bovins de Karin Heegan ne possédant pas le même attribut.

Le style arabo-éthiopien défini par Cervicek (1979) couvre une vaste aire qui comprend la péninsule arabique et l'ensemble de la Corne. Dans ce style, les bovins seraient représentés selon des canons à peu près constants nonobstant les nombreuses variantes : Il s'agit de bovinés sans bosse à longues cornes représentés de profil avec les pattes avant et arrière ne formant que deux lignes épaisses. En revanche le cou et la tête ainsi que les cornes sont vus de derrière ou de dessus. Si l'on s'en tient à ces critères très généraux, il ne fait aucun doute que les bovinés de Las Geel appartiennent à cette famille de style.

Si l'on cherche à comparer nos vaches de Las Geel à celles sculptées ou gravées de la région éthiopienne du Sidamo, on trouvera à la fois des points communs et des différences. Nulle part n'existe à Las Geel ce petit trait vertical à l'extrémité distale des pattes que l'on trouve à Chabbé sur les animaux sculptés en bas relief et qui comme sur les animaux peints de Laga Oda figurent probablement le sabot. C'est pourquoi d'ailleurs nous ne pouvons souscrire à l'opinion récemment exprimée de J.-L. Le Quellec qui propose de faire de l'absence ou de la présence de ce trait un élément discriminant entre le style de Chabbé-Galma et celui de Laga-Oda-Sourré (Le Quellec et Gezachew, 2001). La silhouette générale est traitée dans l'esprit du style de Chabbé-Galma, mais avec quelques différences notables : la croupe est droite alors qu'elle est arrondie à Chabbé et à Galma; la ligne du ventre est concave alors qu'elle est biconcave à Chabbé; les mamelons des pis sont très longs sur les gravures de 
Chabbé et le pis est disproportionné par rapport au volume du corps. A Las Geel, les mamelons sont beaucoup moins "exubérants" qu'à Chabbé et les proportions du pis restent plus compatibles avec le volume du reste du corps. C'est finalement la façon de représenter les cornes qui rapproche le plus les deux styles encore que les vaches de Chabbé présentent souvent une dissymétrie entre la corne droite et la corne gauche qui forment comme une sorte de $\mathrm{L}$.

Quant aux comparaisons que l'on peut tenter de faire avec les bovinés du Harar, on retiendra par exemple la présence des croupes droites dans les deux cas et une forte similitude dans la silhouette générale des animaux. Mais le point de comparaison le plus intéressant, eu égard à la singularité des représentations bovines de Las Geel, reste la volonté d'orner le cou des animaux. Certes, ce trait stylistique apparaît de façon très discrète dans l'art rupestre du Harar de sorte qu'il n'a jamais réellement retenu l'attention des chercheurs. Aujourd'hui, avec la découverte du site de Las Geel, il prend une autre signification. C'est principalement à Laga Oda, mais aussi à Sourré, Goda Dassa, Goda Allele et Ourso (Bouakaze-Khan, 2002) que l'on observe quelques bovinés portant des décorations sur ou au-dessous du cou. Pour celles qui sont figurées sur le cou, il s'agit d'une série de traits superposés rayant le cou en travers à l'image d'une superposition de colliers. Ce sont ces ornementations qui peuvent être rapprochées de nos "plastrons" de Las Geel. Pour les autres, il s'agit d'une ligne d'appendices subrectangulaires accolés placés exactement à l'emplacement de cette partie anatomique très développée chez certaines races bovines actuelles estafricaines et que l'on appelle "les fanons", sorte de frange de peau pendant sous le cou. En dehors des sites à peintures du Harar, on ne trouve pas ces caractères ornementaux dans la Corne de l'Afrique pas plus à Djibouti où on a affaire exclusivement à des gravures (Joussaume, 1987) qu'en Erythrée (Calegari, 1999). Rappelons ici ce que nous avons mentionné plus haut : la similitude entre le bloc peint de l'encolure d'une vache de Karin Heegan et les décors des vaches de Las Geel. Nous ne trouvons pas en revanche à Las Geel le trait flanqué de barbelures reliant les extrémités des deux cornes de la vache de Karin Heegan. Nous ne trouvons pas non plus les petits appendices filiformes ou triangulaires accrochés à la pointe d'une corne, comme à Chabbé ou sur la même vache de Karin Heegan.

\subsection{Le contexte régional}

Nous n'avons vu que quelques abris ornés lors de notre mission de novembre-décembre 2002 au Somaliland. En dehors de l'extraordinaire site de Las Geel, les autres ne possèdent que de modestes œuvres appartenant à des périodes diverses. A l'extrémité occidentale du grand massif calcaire des monts Goolis, près de l'éperon de Gaan Libahh, la grotte 1 de Garba Keyle, fouillée par le major Glover dans les années 1930, a livré des industries lithiques attribuées au Somaliland Wilton par J. D. Clark (Clark 1954, p. 349). A l'entrée de cette grotte se trouvent sur les deux parois des peintures rouges ou noires peu lisibles et stylisées qui ne s'apparentent pas à celles de Las Geel. Il s'agit d'éléphants, de girafes, d'antilopes, peut-être d'un lion et d'un boviné. Des 
gravures postérieures figurant des dromadaires sont visibles sur la paroi de droite en entrant, celle qui comporte la plupart des animaux peints.

Les abris inédits de Daymolhe ou de Dhagaxa Sumadaha Le au sud de Berbera ne possèdent que des signes tribaux peints en rouge ou en noir et d'âge probablement récent. Certes, d'autres sites ornés importants ont été signalés lors de diverses missions européennes ou américaines dans l'ancienne Somalie britannique ou italienne, mais les représentations graphiques ou les photographies qu'en donnent Clark ou d'autres auteurs sont peu nombreuses. Dans sa thèse (op.cit.), D. Bouakaze-Khan recense 21 sites ornés sur l'ensemble de la Somalie et du Somaliland et ne peut guère reproduire que quelques planches de signes tribaux pour la plupart provenant de l'étude réalisée par S.A. Brandt en Somalie centrale dans les abris s'ouvrant sur les flancs de l'inselberg de Buur Heybe déjà étudié par Graziozi puis par Clark. Il reproduit aussi les dessins des panneaux de Karin Heegan déjà publiés par Brandt (Brandt et Carder, 1987). Nous ajouterons en dernier lieu à ce maigre tour d'horizon régional le rocher gravé de Hor Jog sur le flanc oriental du plus haut sommet du massif du Wagar, à une cinquantaine de $\mathrm{km}$ à l'est de Sheikh. C'est un petit bloc de moins d'un $\mathrm{m}^{3}$ qui porte sur sa face supérieure 2 bovinés gravés à dos plat et cornes en pince, sans mamelles, et qui mis à part ce détail significatif, ont une silhouette et des proportions proches de celles des bovinés sculptés en champlevé de Galma dans le Sidamo (Joussaume et al.,1994).

\section{Conclusion et perspectives}

La découverte du site de Las Geel le 4 décembre 2002 fut pour notre équipe un moment intense d'émotion tant il est vrai que cet ensemble de 10 abris ornés est exceptionnel à la fois par la qualité, l'originalité et l'état de conservation de la plupart de ces œuvres peintes sur le rocher. Nous n'avons pu passer que quelques heures sur le site et de plus, une partie des abris n'a pu être photographiée en raison de la présence d'essaims de grosses guêpes très agressives. C'est notamment le cas d'un des deux abris situés le plus au Nord et qui, vu d'une certaine distance et à l'aide de jumelles semble particulièrement riche en figurations animales remarquablement bien conservées. Une fois cette question résolue, nous espérons accéder en toute quiétude à l'ensemble de ces abris et en réaliser une étude détaillée. C'est l'objectif que nous nous sommes fixé avec l'accord des autorités somalilandaises pour une prochaine mission fin 2003. Dans cette attente, nous avons encouragé le ministère de la culture et du tourisme à prendre des mesures de surveillance du site.

Le site de Las Geel est certainement l'un des plus exceptionnels de la Corne de l'Afrique. Le traitement particulier des bovinés femelles qu'il offre peut à lui seul en faire le site éponyme d'un nouveau style d'art rupestre qu'il reste à situer dans le temps par rapport aux autres ensembles régionaux déjà décrits (Djibouti, Erythrée, Ethiopie) sans oublier le Yemen et l'Arabie saoudite.

Les nombreux massifs rocheux propices à ce mode d'expression artistique qui parsèment le territoire de la République de Somaliland ont en fin de compte 
été très peu prospectés depuis que les chercheurs occidentaux se sont intéressés à ces contrées. Il est donc probable que de nouvelles découvertes permettront dans les années à venir de mieux connaître cet art pré ou protohistorique.

\section{BIBLIOGRAPHIE}

Bouakaze-Khan 2002 : BOUAKAZE-KHAN (D.). L'art rupestre de la Corne de l'Afrique. Etude globale dans son contexte archéologique et anthropologique. Modèle d'interprétation. Thèse, univ. de Paris 1 Panthéon-Sorbonne, Paris, 2002. 2 vol. de 216 et $356 \mathrm{p}$.

Brandt 1986 : BRANDT (S. A). "The Upper Pleistocene and early Holocene prehistory of the Horn of Africa", The African Archaeological Review, 4, 1986, p. 41-82.

Brandt 1989 : BRANDT (S. A.). "L'Age de la Pierre en Somalie", L'Anthropologie, Paris, 94, 1989, 3, p. 459-482.

Brandt et Carder 1987 : BRANDT (S. A.) and CARDER (N.). "Pastoral rock art in the Horn of Africa : Making sense of udder chaos", World Archaeology, 19, 2, 1987, p. 194-213.

Calegari 1999 : CALEGARI (J.). L'arte rupestre dell'Eritrea. Repertorio ragionato ed esegesi iconografica. Memorie delle Società Italiana di Scienze Naturali e del Museo Civico di Storia Naturale di Milano, vol. XXIX, fasc. 1, Milano, 1999. $174 \mathrm{p}$.

Cervicek 1979 : "Some African affinities of Arabian rock art", Rassegna di studi etiopici, XXVII, 1978-79, p. 5-12.

Clark 1954 : CLARK (J. D.). The prehistoric cultures of the Horn of Africa. Cambridge University Press, 1954,374 p., 52 pl. et 3 tab. h.t.

Joussaume 1981 : JOUSSAUME (R.). "L'art rupestre de l'Ethiopie", in : Hommage au professeur L. Balout. Synthèse $n^{\circ} 6$ 6. 1981, p. 159-175.

Joussaume 1987 : JOUSSAUME (R.). "Gravures rupestres en République de Djibouti", Bull. du Groupe vendéen d'études préhistoriques, 17, 1987, p. 2445.

Joussaume 1989 : JOUSSAUME (R.). "Gravures rupestres en République de Djibouti”, Bull. de l'ISERST, Djibouti, n², p. 105-129.

Joussaume dir. 1995 : JOUSSAUME (sous la dir. de R.). Tiya-L'Ethiopie des mégalithes. Du biface à l'art rupestre dans la Corne de l'A frique. Association des publications chauvinoises (A.P.C), Chauvigny, 1995. 387 p., 375 fig.

Joussaume et al. 1994 : JOUSSAUME (R.)., BARBIER (S.) et GUTHERZ (X.), "L'art rupestre du Sidamo (Ethiopie)", International Newsletter on Rock Art, 9, 1994, p. 7-11.

Le Quellec et Gizachew 2001 : LE QUELLEC (J.-L.) and GIZACHEW ABEGAZ, "New sites of South Ethiopian rock engravings : Godana Kinjo, Ejersa Gara Hallo, and remarks on the Sappe-Galma school", Annales d'Ethiopie, XVII, 2001, p. 205-224. 


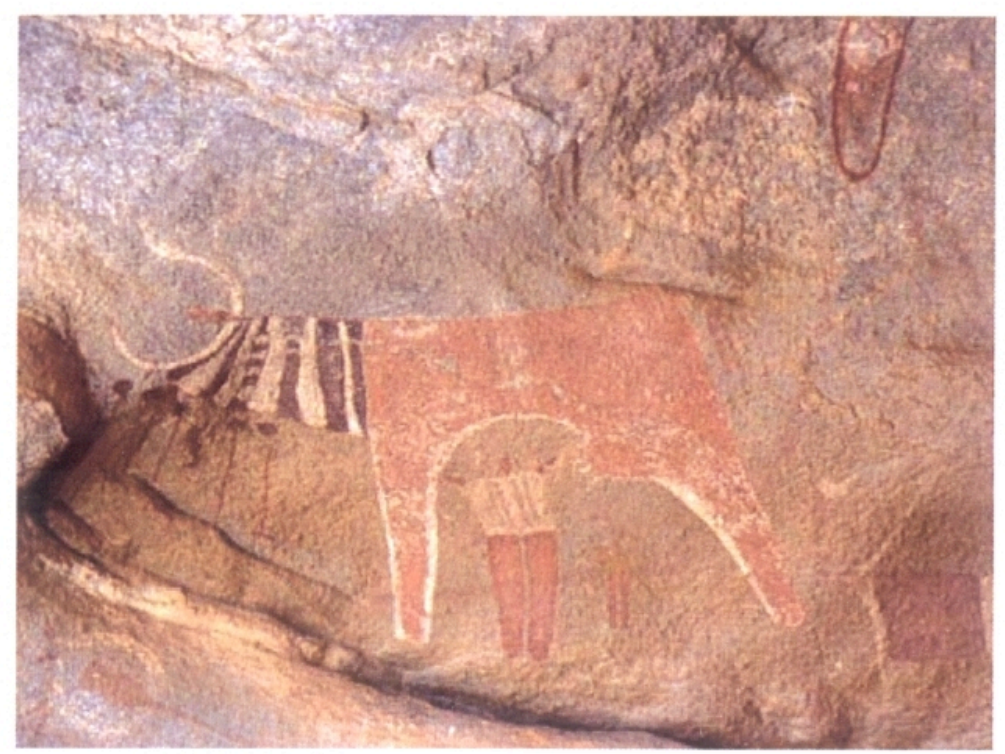

Planche 1

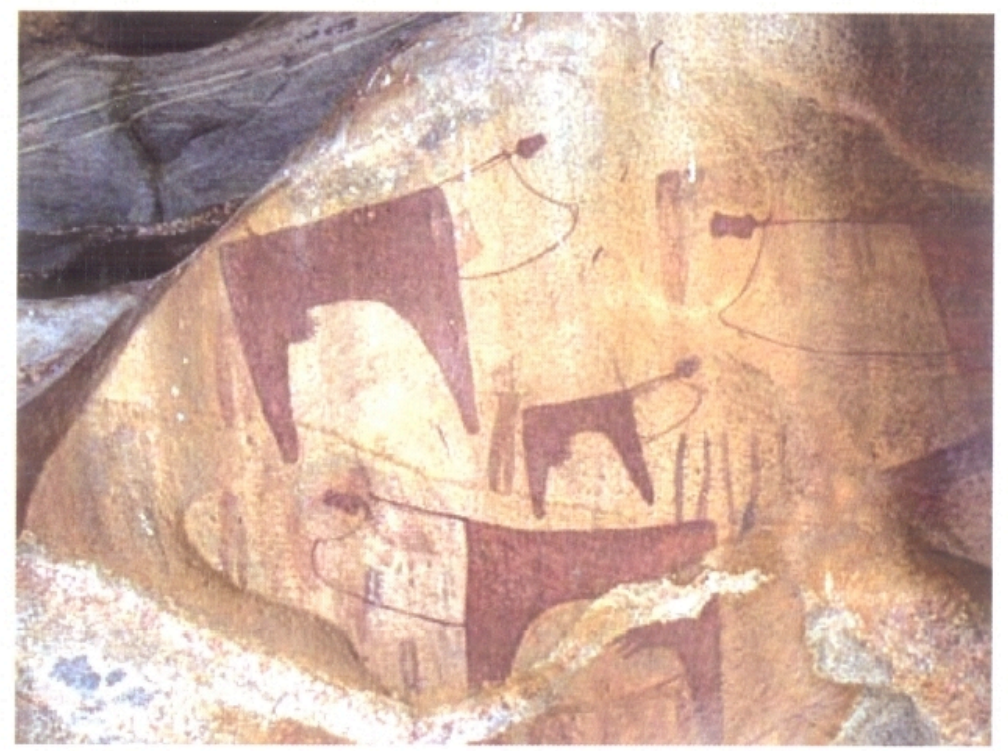

Planche 2

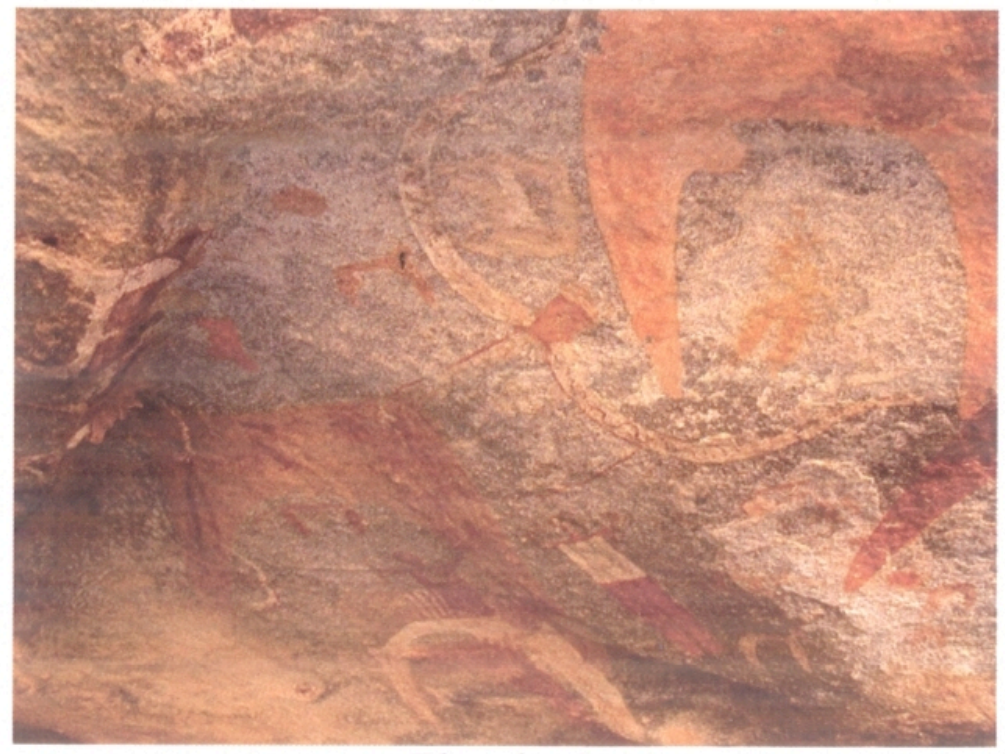

Planche 3 


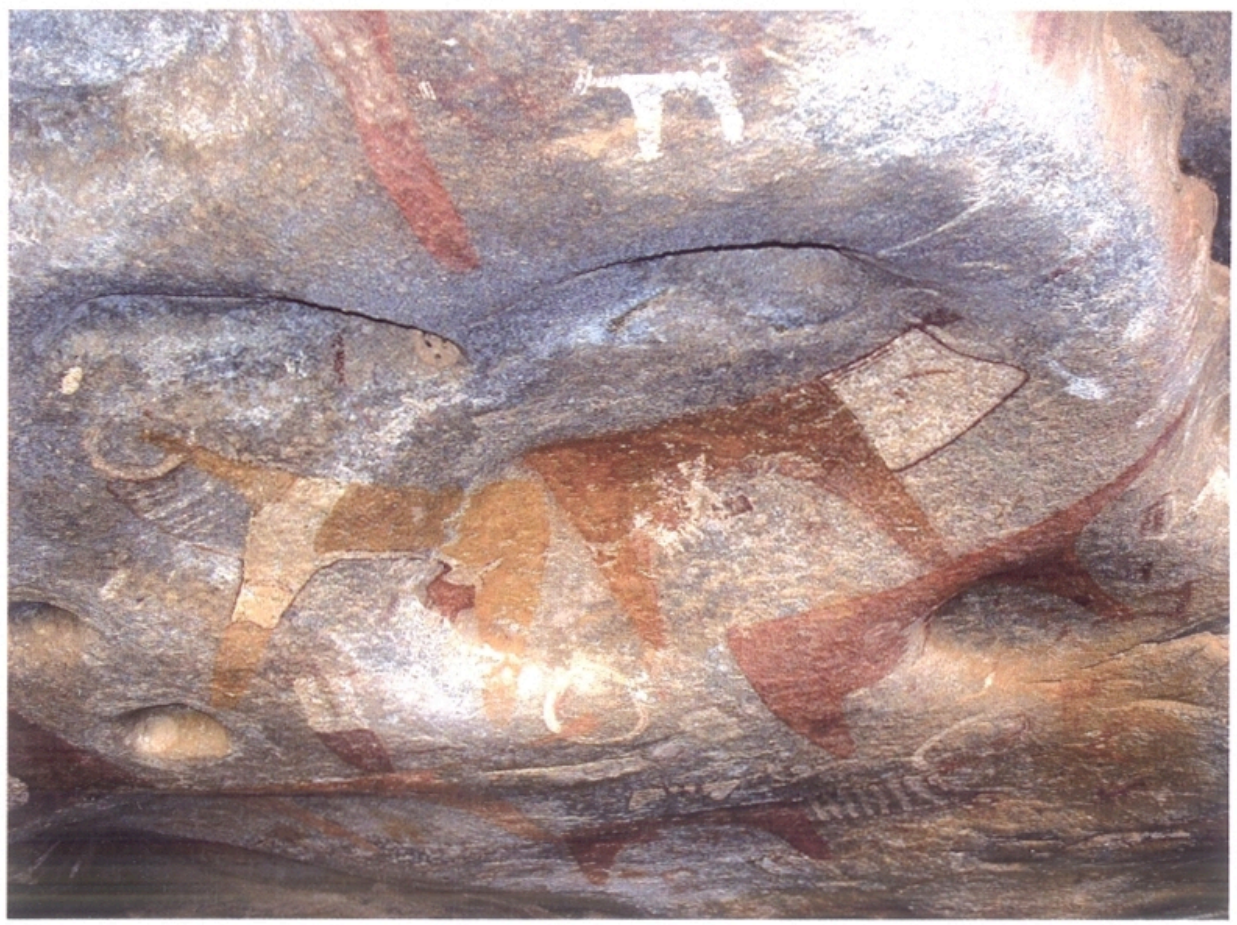

Planche 4

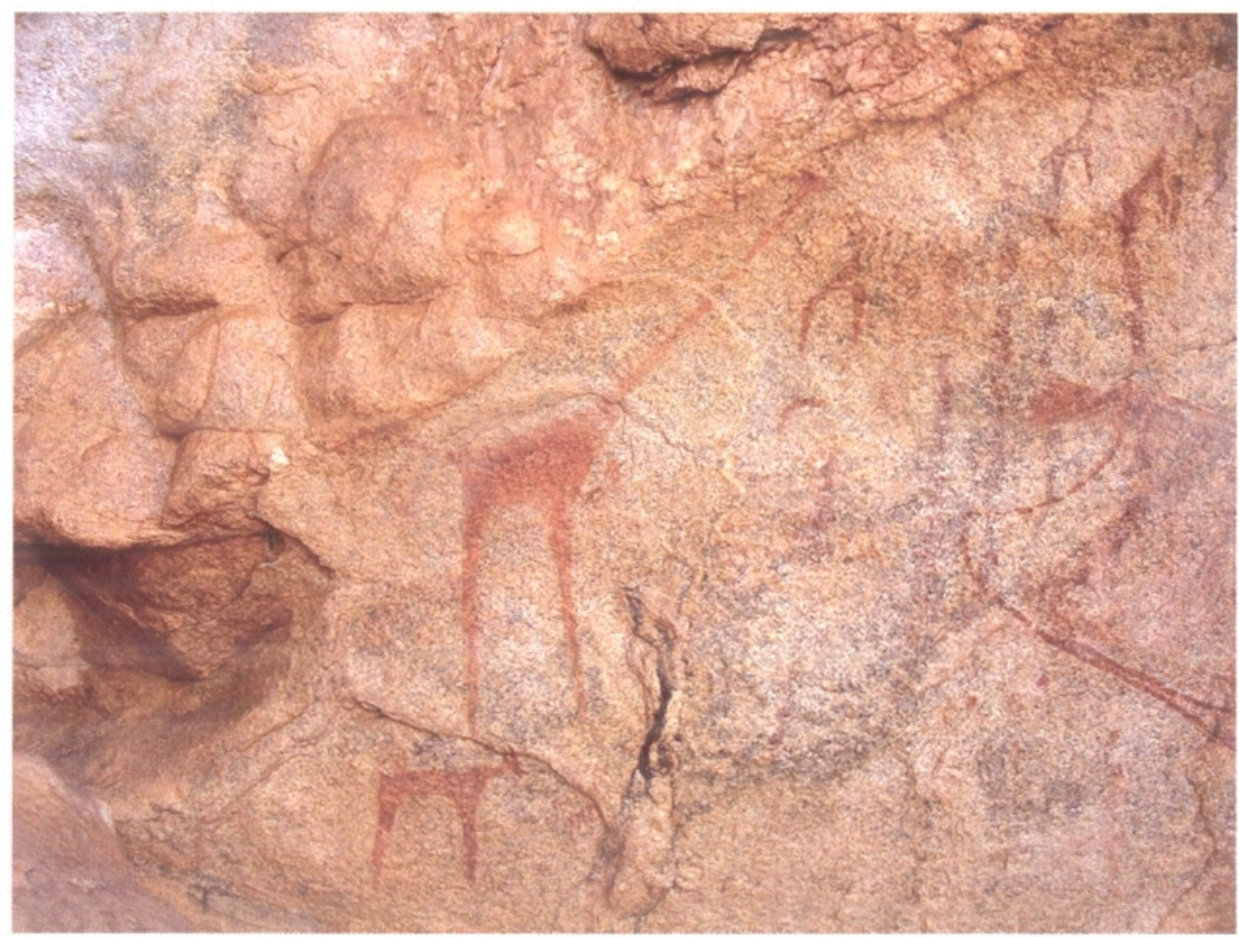

Planche 5 